\title{
Programa de trastornos de alimentación en adolescentes y jóvenes chilenos: variaciones a seis meses de tratamiento
}

\author{
Patricia Cordella M, Paula Lizana Ca, Pascuala U rrejola $\mathbf{N}$, \\ Dolly Figueroa E, Pilar del Río V, Isabel Hodgson B, \\ Consuelo San Martín Ba, Carol U nger $\mathrm{Ch}^{\mathrm{a}}$.
}

Multidisciplinary treatment of eating
disorders in Chilean adolescents
and young adults. Six months outcome

Background: Eating disorders may have serious organic consequences derived from under nutrition, specific nutrient deficiencies and electrolytic disturbances and reach a mortality as high as $12 \%$. Aim: To describe the features and outcome after six months of treatment of patients attending the Eating Disorders Unit at the Catholic University Hospital in Chile. Material and methods: Review of medical records of patients with eating disorders that received a multidisciplinary treatment by a team of nutritionists, psychiatrists and psychologists and were seen at least twice in a period of six months. Results: The records of 81 patients (mean age 16.3 \pm 3 years, only one male) were included in the review. Forty nine patients had Anorexia Nervosa of the restricting type, five of the purging type, 22 had Bulimia Nervosa of the purging type and two of the non purging type and three patients had an eating disorder not otherwise specified. Eighty percent consulted within the first year of symptom appearance. Sixty five percent came from families with both parents living together, $57 \%$ had a rigid and agglutinated family structure and $65 \%$ had occult crises. Depression or dysthymia was found in $45 \%$ of patients and drug therapy was required at the beginning of treatment in $25 \%$. Obsessive traits (40.4\%) were significantly related to restrictive eaters. A past history of obesity or overweight was common. After six months of treatment, body mass index increasing significantly in restrictive eaters with obsessive traits and occult family crises was noticed. Conclusions: A multidisciplinary therapeutic intervention improved body mass index and family symptoms in patients with restrictive eating disorders (Rev Méd Chile 2006; 134: 973-80). (Key w ords: Adolescent; Anorexia nervosa; Bulimia nervosa; Eating disorders)

Recibido el 12 de julio, 2005. Aceptado el 2 de enero, 2006.

Programa Trastornos de Alimentación, Pontificia Universidad Católica de Chile. Departamentos de Psiquiatría y Pediatría, Escuela de Medicina, Pontificia Universidad Católica de Chile. aPsicóloga - Terapeuta Familiar

Correspondencia a: Dra. Patricia Cordella. La Capitanía 976,

Las Condes, Santiago. E mail: cordella@mi.cl 
T os trastornos de alimentación son un tema de Lran interés, tanto para los especialistas como para el público general, pues se trata de una particular clínica que nos deja perplejos; ¿cómo comprender una patología que parece generarse desde la voluntad y que, sin aparente control, se consolida tanto en alteraciones mentales, como orgánicas?

Se trata de un trastorno psíquico que genera consecuencias orgánicas graves ${ }^{1-4}$, derivadas de la desnutrición, la restricción y los desequilibrios hidroelectrolíticos, alcanzando una mortalidad de $12 \%$, tanto por causas médicas como siquiátri$\operatorname{cas}^{5,6}$

El DSM IV ${ }^{7}$ clasifica los trastornos de la conducta alimentaria en:

1. Anorexia nerviosa: restrictiva, compulsiva y purgativa. 2. Bulimia nerviosa: purgativa, no purgativa. 3. Trastornos de la conducta alimentaria no especificado. Además, tanto la conducta alimentaria como la función de la imagen corporal en la identidad, serían alteraciones comunes a todos ellos.

Hablar de trastornos de alimentación (TAL) amplía el concepto de la patología, no sólo hacia las conductas evidentes, sino a las dinámicas psíquicas subyacentes. La solución anoréxica ${ }^{8}$ se instala dentro de un sistema fisiológico vulnerable al estrés, que se ha desarrollado dentro de un sistema vincular de fallas y faltas reiteradas ${ }^{9,10}$.

En la etiología de los trastornos de alimentación se encuentran interactuando factores propios del sujeto como: a. la genética ${ }^{11}$; b. la inestabilidad del eje hipotálamo-pituitaria-adrenal ${ }^{12}$, resultado de deficiencias en la contención de las angustias desencadenadas en eventos vitales traumatizantes $^{13-15}$; c. el género ${ }^{16}$; d. las dinámicas familiares y sociales que estructuran la voluntad y el deseo ${ }^{17}$. Por otra parte, la desnutrición aguda 0 los desequilibrios hidroelectrolíticos facilitan $\mathrm{y}$ consolidan alteraciones psíquicas ${ }^{18-21}$.

La familia parece cumplir un rol esencial en los cambios necesarios para revertir el fenómeno psíquico a la base $22-24 \mathrm{y}$, por otra parte, el trabajo en equipo (que incluye tanto intervenciones nutricionales, como psicoterapéuticas y psiquiátricas) ha probado ser un método eficiente de intervención si está basado en un modelo coherente y compartido de comprensión de la patología anoréxica ${ }^{13,14,25}$.
Entre los indicadores de cambio, el índice de masa corporal (IMC) es ampliamente utilizado para evaluar el estado nutricional. Esto, por su relación con la grasa corporal total. Aun variando edad y madurez biológica es capaz de describir los cambios en la composición corporal que derivan de los cambios en la conducta alimentaria.

La intervención familiar modifica patrones conductuales y emocionales que sostienen la patología, por esto, otro indicador de cambio es la capacidad familiar de asumir problemas, es decir, enfrentar $\operatorname{conflictos}^{26}$ abriendo las crisis a su comprensión y resolución.

Otra medición utilizada para medir cambio terapéutico ha sido el eating disorders inventory (EDI), con 11 subescalas de funcionamiento social, personal y corporal relacionados con los trastornos de alimentación ${ }^{27,28}$.

El objetivo de este estudio fue caracterizar una muestra de adolescentes y jóvenes consultantes a un programa de trastornos de alimentación, y describir los cambios orgánicos y familiares después de seis meses de intervención, de acuerdo con el tipo de trastorno de alimentación a través del registro clínico de datos psiquiátricos, familiares y nutricionales.

\section{MATERIAL Y MÉTODO}

Del total de registros de ingresos psiquiátricos entre 1998 y 2003 (n: 144) al programa de trastornos de alimentación de la Pontificia Universidad Católica de Chile, se seleccionan 81 pacientes que recibieron atención multidisciplinaria, definida como dos o más contactos con cada una de tres instancias: nutrición, psiquiatría y terapia psicológica, desde su ingreso y hasta seis meses después.

En la consulta siquiátrica se evaluó, a través de entrevista clínica, los ejes de la patología mental. En el Eje I, los síndromes psiquiátricos propiamente tales; en el Eje II la personalidad, en el Eje III enfermedades médicas, en el Eje IV el funcionamiento social y familiar, y en el Eje V, el ajuste biopsicosocial al estrés. Se clasifican según criterios del $\mathrm{DSM} \mathrm{IV}^{7}$.

En la consulta nutricional se incluyó determinación de peso y talla con una balanza de precisión con cartabón incluido (SECA), con una sensibilidad 
de $100 \mathrm{~g}$ y $1 \mathrm{~mm}$, respectivamente. Cada paciente fue medido y pesado en ropa interior. El IMC fue calculado dividiendo el peso, expresado en kilos por el cuadrado de la estatura expresada en metros (peso/talla2). En las menores de 20 años se utilizaron tablas CDC de IMC con su respectivo puntaje $\mathrm{z}$, usando como referencia las curvas de crecimiento CDC-NCHS 1997, e IMC expresado como score z (IMCZ=IMC real-IMC mediana/1 DS), usando como referencia tablas locales ${ }^{29}$.

En la consulta terapéutica la evaluación se realizó con el paciente y su familia utilizando el modelo circunflejo de Olson ${ }^{30}$, que clasifica a las familias según su organización en: 1. adaptabilidad (capacidad de cambio ante diversas circunstancias): rígidas o caóticas y 2. cohesión (manejo de las distancias emocionales): aglutinadas y desligadas. El examen clínico de las relaciones familiares permite reconocer, además, el conflicto en abierto y cerrado, es decir, explicitado o encubierto en la historia contada por la familia.

Estadística. El procedimiento de comparación utilizó tablas de contingencia entre las variables cuya asociación pudiese resultar relevante. Se determinó la significación de la asociación con la prueba de Chi cuadrado $\left(\chi^{2}\right)$ de Pearson y se evaluó el cambio en la proporción de familias con crisis abierta y cerrada a través de la prueba de McNemar. Una probabilidad $<0,05$ rechazó la hipótesis de nulidad. También se evaluó la evolución del IMC en puntajes z al inicio $\mathrm{y}$ a los seis meses mediante prueba $\mathrm{t}$ de student para mediciones repetidas. Se utilizó promedio \pm desviación estándar.

\section{RESULTADOS}

El promedio de edad al consultar por primera vez fue de 16,3 \pm 3,0 años (rango 10-26 años). La edad promedio de inicio del trastorno alimentario fue $15,2 \pm 2,1$ años. El tiempo de evolución del trastorno alimentario previo a la consulta fue de $1,4 \pm 2,1$ años. En la muestra sólo hay un adolescente varón de 14 años con diagnóstico de anorexia restrictiva.

La Tabla 1 muestra la distribución diagnóstica al ingreso. Se diagnosticó anorexia restrictiva en $60,5 \%(n=49)$. Los pacientes con anorexia restrictiva se concentraban entre los 12 y 18 años (n: 40) y aquellos con bulimia purgativa se encontraban entre los 14 y 22 años (n: 21). Durante el período estudiado, 6 pacientes restrictivas requirieron hospitalización por causas nutricionales, en hospital general. De las 48 pacientes restrictivas, 23 presentaban amenorrea. El 12,2\% (n: 6) tenía un IMC $<15$, mientras $51 \%$ tenía un IMC entre 15 y 17,9 al ingreso.

En la Tabla 2 se compara el IMC en puntaje $\mathrm{z}$ al inicio y a los 6 meses de tratamiento en las pacientes menores o igual a 20 años. Hay diferencias significativas para el grupo de pacientes con anorexia restrictiva $(\mathrm{t}=-2,559 ; \mathrm{gl}=29 ; \mathrm{p}=0,016)$, mientras las pacientes con bulimia purgativa se mantuvieron relativamente estables en el IMC ( $t=-0,776, g l=6, p=0,467)$.

En la Tabla 3 se describe la relación entre obesidad o sobrepeso y el tipo de trastorno de alimentación. Treinta y siete por ciento de los pacientes tenía antecedentes asociados al trastomo alimentario al ingreso $\left(\chi^{2}=11,36 ; \mathrm{gl}=4 ; \mathrm{p}=0,023\right)$.

Tabla 1. D istribución de frecuencias de edad al consultar según tipo de trastorno alimentario al ingreso

\begin{tabular}{|lrrrrrrr|}
\hline Epc & AR & BP & BNP & AP & TNE & Total & $\%$ \\
& N:49 & N:21 & N:2 & N:5 & N:3 & N:81 & \\
\hline$\leq 12,0$ & 4 & 0 & 0 & 0 & 0 & 4 & 12,1 \\
$12,1-14,0$ & 0 & 0 & 0 & 2 & 1 & 14 & 17,3 \\
$14,1-16,0$ & 19 & 8 & 0 & 0 & 1 & 28 & 34,6 \\
$16,1-18,0$ & 11 & 6 & 1 & 1 & 1 & 20 & 24,7 \\
$18,1-20,0$ & 2 & 4 & 1 & 1 & 0 & 8 & 9,9 \\
$20,1-22,0$ & 1 & 3 & 0 & 0 & 0 & 4 & 4,9 \\
$22,1-24,0$ & 0 & 0 & 0 & 1 & 0 & 1 & 1,2 \\
$24,1+$ & 2 & 0 & 0 & 0 & 0 & 2 & 2,5 \\
\hline
\end{tabular}

Epc: edad primera consulta. AR: anorexia restrictiva. BP: Bulimia restrictiva. BNP: Bulimia no purgativa. AP: anorexia purgativa. TNE: Trastorno no específico. 
Los rasgos de personalidad según el tipo de TAL aparecen en la Tabla 4. Los pacientes con anorexia restrictiva se asociaron a rasgos de personalidad obsesiva $\left(\chi^{2}=38,084 ; g l=24, p=0,034\right)$.

La comorbilidad más frecuente fueron los trastornos afectivos (depresión mayor, distimia), $47,5 \%$ en la anorexia restrictiva y $22,5 \%$ en la bulimia purgativa. El tratamiento fue iniciado sin indicación de psicofármacos en $75,3 \%$ de los pacientes, mientras $14,8 \%$ fue tratado con antidepresivos desde el inicio, frecuentemente inhibidores de la recaptura de la serotonina (IRS), benzodiazepinas y, en dos casos, antipsicóticos como risperidona y fluxipentol.

Tabla 2. Comparación del IM C en puntaje $Z$ al inicio y a los seis meses según tipo de trastorno alimentario diagnosticado al ingreso al tratamiento (edad $\leq 20$ años)

\begin{tabular}{|lcc|}
\hline Tipo de trastorno alimentario & $\begin{array}{c}\text { IMC inicio } \\
(\mathrm{Z})\end{array}$ & $\begin{array}{c}\text { IMC } 6 \text { meses } \\
(\mathrm{Z})\end{array}$ \\
\hline Anorexia restrictiva & $-0,68 \pm 0,64$ & $-0,42 \pm 0,42^{*}$ \\
Bulimia purgativa & $-0,15 \pm 0,67$ & $-0,03 \pm 0,66$ \\
Bulimia no purgativa & $1,83 \pm 0,95$ & $1,35 \pm 0,98$ \\
Anorexia purgativa & $-0,20 \pm 0,49$ & $0,25 \pm 0,2$ \\
Trast. no especificado & $-0,36 \pm 0,3$ & $-0,2 \pm 0,42$ \\
& $-0,44 \pm 0,81$ & $-0,23 \pm 0,6$ \\
\hline
\end{tabular}

$*_{t}=-2,559 . p=0,016 . g l=29$.

Tabla 3. Tabla de contingencia entre antecedentes de sobrepeso u obesidad según tipo de trastorno alimentario diagnosticado al ingreso

\begin{tabular}{|c|c|c|c|c|c|c|c|c|c|c|c|c|}
\hline ASPO & \multicolumn{2}{|c|}{$\mathrm{AR}$} & \multicolumn{2}{|c|}{ BP } & \multicolumn{2}{|c|}{ BNP } & \multicolumn{2}{|c|}{$\mathrm{AP}$} & \multicolumn{2}{|c|}{ TNE } & \multicolumn{2}{|c|}{ Total } \\
\hline $\mathrm{N}: 81$ & $\mathrm{~N}$ & $\%$ & $\mathrm{~N}$ & $\%$ & & $\%$ & $\mathrm{~N}$ & $\%$ & & $\%$ & & \\
\hline Con antec & 37 & 45,6 & 10 & 12,3 & 0 & & 3 & 3,7 & 1 & 1,2 & 51 & 62,9 \\
\hline Sin antec & 12 & 14,8 & 12 & 14,8 & 2 & 2,4 & 2 & 2,4 & 2 & 2,4 & 30 & 37,3 \\
\hline Total & 49 & 60,4 & 22 & 27,1 & 2 & 2,4 & 5 & 6,1 & 3 & 3,7 & $81 *$ & 100 \\
\hline
\end{tabular}

$\chi^{2}=11,36 . g l=4 . p=0,023$. ASPO: antecedentes de sobrepeso y/o obesidad. Epc: edad primera consulta. AR: anorexia restrictiva. BP: Bulimia restrictiva. BNP: Bulimia no purgativa. AP: anorexia purgativa. TNE: trastorno no especificado.

Tabla 4. Tabla de contingencia para rasgos de personalidad según tipo de trastorno alimentario diagnosticado al ingreso

\begin{tabular}{|c|c|c|c|c|c|c|c|c|c|c|c|}
\hline \multirow[t]{2}{*}{ TRP } & \multicolumn{2}{|c|}{ AR } & \multicolumn{2}{|c|}{ BP } & \multicolumn{2}{|c|}{ BNP } & \multicolumn{2}{|c|}{$\mathrm{AP}$} & \multicolumn{2}{|c|}{ TNE } & \multirow{2}{*}{$\begin{array}{c}\text { Total } \\
(\%)\end{array}$} \\
\hline & $\mathrm{N}: 35$ & $(\%)$ & $\mathrm{N}: 10$ & $(\%)$ & $\mathrm{N}: 2$ & (\%) & $\mathrm{N}: 3$ & $(\%)$ & $\mathrm{N}: 2$ & $(\%)$ & \\
\hline Límite & 3 & $(5,7)$ & 7 & (14) & 2 & $(3,8)$ & 0 & & 0 & & 23,5 \\
\hline Dependiente & 4 & $(7,8)$ & 1 & $(1,9)$ & 0 & & 0 & & 0 & & 9,7 \\
\hline Depend-límite & 0 & & 1 & $(1,9)$ & 0 & & 0 & & 0 & & 1,9 \\
\hline Obsesiva & 14 & (27) & 0 & 0 & 0 & & 1 & $(1,9)$ & 2 & $(3,8)$ & $32,7 *$ \\
\hline Obsesiva-límite & 3 & $(5,7)$ & 0 & 0 & 0 & & 1 & $(1,9)$ & 0 & & 7,6 \\
\hline Obsesiva-evitativa & 11 & $(21,1)$ & 1 & $(1,9)$ & 0 & & 1 & $(1,9)$ & 0 & & 24,9 \\
\hline
\end{tabular}

* $\chi^{2}$ 38,084. gl=24. $p=0,034$. TPR: tipo rasgo personalidad. Epc: edad primera consulta. AR: anorexia restrictiva. BP: bulimia restrictiva. BNP: bulimia no purgativa. AP: anorexia purgativa. TNE: trastorno no especificado. 


\section{Tabla 5. Tabla de contingencia para tipo de familia (aglutinamiento/rigidez) según tipo de trastorno alimentario diagnosticado al ingreso}

\begin{tabular}{|c|c|c|c|c|c|c|c|c|c|c|c|c|}
\hline \multirow[t]{2}{*}{$\mathrm{TF}$} & \multicolumn{2}{|c|}{ AR } & \multicolumn{2}{|c|}{ BP } & \multicolumn{2}{|c|}{ BNP } & \multicolumn{2}{|c|}{ AP } & \multicolumn{2}{|c|}{ TNE } & \multicolumn{2}{|c|}{ Total } \\
\hline & $\mathrm{N}: 49$ & (\%) & $\mathrm{N}: 22$ & (\%) & $\mathrm{N}: 2$ & (\%) & $\mathrm{N}: 5$ & (\%) & $\mathrm{N}: 3$ & (\%) & $\mathrm{N}: 81$ & (\%) \\
\hline FAR & 28 & $(34,5)$ & 11 & $(13,5)$ & 1 & $(1,4)$ & 4 & $(4,9)$ & 2 & $(2,5)$ & 46 & $(56,7)$ \\
\hline FAF & 7 & $(8,6)$ & 2 & $(2,4)$ & 0 & & 0 & & 0 & & 9 & $(11,1)$ \\
\hline FDR & 7 & $(8,6)$ & 7 & $(8,6)$ & 1 & $(1,4)$ & 0 & & 1 & $(1,6)$ & 16 & $(12,3)$ \\
\hline FDF & 2 & $(2,4)$ & 1 & $(1,4)$ & 0 & & 1 & $(1,4)$ & & & 4 & $(4,9)$ \\
\hline SDI & 5 & $(6,1)$ & 1 & $(1,4)$ & 0 & & 0 & & 0 & & 6 & $(7,4)$ \\
\hline
\end{tabular}

${ }^{*} \chi^{2}=12,53 ; g l=16 ; p=0,707$. FAR: Aglutinada-rígida. FAF: Aglutinada flexible. FDR: desligada rígida. FDF: desligada flexible. SDI: sin diagnóstico al ingreso. AR: anorexia restrictiva. BP: bulimia restrictiva. BNP: bulimia no purgativa. AP: anorexia purgativa. TNE: trastorno no específico.

Por otra parte, en la Tabla 5, se muestra que $65,4 \%$ de las familias eran biparentales (ambos padres a cargo), siendo el resto separados o reconstituidos. Se observó una tendencia de las familias consultantes a ser aglutinadas y rígidas. Sin embargo, no se observó asociación estadísticamente significativa entre el tipo de familia y el diagnóstico de trastornos de alimentación al ingreso $\left(\chi^{2}=12,53 ; \mathrm{gl}=\right.$ 16 ; $p=0,707$ y $\chi^{2}=4,593 ; g l=8 ; p=0,8$, respectivamente). Además, no se observó asociación estadísticamente significativa entre el tipo de crisis familiar (crisis abierta versus crisis cerrada) y el diagnóstico al ingreso $\left(\chi^{2}=1,691 ; g=4 ; p=0,792\right)$.

La proporción de familias (Tabla 6) que cambiaron el manejo de las crisis de conflictos cerrados a conflictos abiertos, fue estadísticamente significativo $(p=0,0001)$. De $73,6 \%$ que comenzó con conflictos cerrados bajó a $51,4 \%$ y de $26,4 \%$ que se inició con conflictos abiertos, aumentó a $48,6 \%$. De modo que, en los primeros seis meses, el terapeuta familiar pudo percibir cambios importantes en la familia.

\section{DISCUSIÓN}

La distribución por sexo (mujeres : hombres) de esta muestra (80:1) no corresponde a la descrita en la literatura, 9:131. Es posible que en nuestro medio se subdiagnostiquen los TAL en varones y sean menos referidos a especialistas.

La población estudiada es una muestra joven (10-26 años) que consulta en el curso del primer año de las manifestaciones clínicas de la enferme-
Tabla 6. Tabla de contingencia para cambios de conflictos familiares de abiertos a cerrados a 6 meses de tratamiento en terapia familiar

\begin{tabular}{|lllll|}
\hline Conflictos & $\mathrm{N}$ & $(\%)$ & $\mathrm{N}$ & $(\%)$ \\
\hline Abiertos & 22 & 26,4 & 39 & 48,6 \\
Cerrados & 59 & 73,6 & 42 & 51,4 \\
\hline
\end{tabular}

dad, en $80,3 \%$. Ambos factores, tanto la precocidad en consultar, como la adolescencia temprana, son reconocidos índices de buen pronóstico en la cura $^{32}$ y podrían estar asociados tanto a una parentalidad alerta, como a un tejido cerebral que se presentaría aún plástico ${ }^{33}$ y sin los estragos de la desnutrición crónica ${ }^{34}$, conservando una adecuada adaptación al estrés y, por lo tanto, con posibilidades de cambio y mejoría.

El elevado porcentaje de anorexia restrictiva $(60,5 \%)$ versus el de bulimia purgativa $(27,2 \%)$, es característico de una población de adolescentes que se inician en los trastornos de alimentación. El cuadro comienza frecuentemente con sobrecontrol de la ingesta ${ }^{35}$, restringiendo cantidad y calidad hasta un momento en que afloja el control. El descontrol gatillaría síntomas bulímicos.

Por otra parte, el hecho que la primera consulta ocurra antes de presentar amenorrea 0 irregularidad menstrual, es índice de precocidad de consulta médica. Sólo 38,3\% de los pacientes tenía amenorrea al ingreso. Esta conducta parental de anticipación corresponde a exitosos resultados 
obtenidos por campañas de promoción de salud adolescente en colegios y medios.

El antecedente de obesidad o sobrepeso está relacionado con la aparición de cualquier trastorno de alimentación ${ }^{11,20,22}$. Este antecedente es aún más relevante en la población nacional, por el aumento de la obesidad ${ }^{36}$ y aparece en $37 \%$ de los pacientes estudiados. Las alteraciones en la conducta del comer se asocia a distorsiones preceptuales básicas, como el hambre y la saciedad y a otras más complejas como la imagen corporal y la función psicosocial ${ }^{37}$. Estos son marcadores de seguimiento en los TAL y es posible que comiencen a alterarse desde el sobrepeso, indicando a éste como el momento oportuno de una intervención preventiva.

Los rasgos de personalidad son los modos utilizados para articularse socialmente y mediarían, también, el intercambio entre el paciente y el equipo ${ }^{26}$, componiendo la relación terapéutica. El perfeccionismo ha sido asociado a los rasgos obsesivos $^{38}$, presentándose en diferentes personalidades como un factor que, a corto plazo, predice síntomas restrictivos junto con la autoevaluación negativa 27,39

En esta muestra, la frecuencia de los rasgos obsesivos en anorexia restrictiva se asocia al incremento del IMC a seis meses. Pensamos que este mismo rasgo le permite al paciente vencer el temor a subir de peso, siendo perfeccionista en el seguimiento de las indicaciones nutricionales. Cumplir con proporciones, horarios y selección de alimentos son modos homólogos a los mecanismos obsesivos que han sido activados durante la restricción. Así, el propio sistema que impulsa al paciente a la patología es redirigido hacia la cura. Por otra parte, los padres son llamados a participar como acompañantes durante las comidas, modificando así hábitos interaccionales cotidianos y posibilitando reacomodos en las pautas de relación familiar que sostendrían la patología. La sinergia producida por estos cambios en el sistema de cuidados del paciente, podrían dar cuenta del incremento de peso inicial. Sin embargo, el tratamiento de los trastornos de alimentación es una apuesta a largo plazo y se requieren estudios de seguimiento para medir la estabilidad de los cambios.

La comorbilidad más frecuente en los TAL es el trastorno afectivo, tal como se observa en estudios de metaanálisis ${ }^{40}$. Es posible que los cambios metabólicos asociados a la restricción alimentaria y al vómito, participen en modular los equilibrios emocionales y, por otra parte, los cambios de ánimo ligados al ambiente, condicionen excesos o restricciones de alimentos. Se instalaría así un sistema reverberante emociónconducta alimentaria.

$\mathrm{Si}$ esto ocurre en fenotipos vulnerables, es decir ejes hipotálamo-pituitaria-adrenal que han sido sensibilizados al estrés tras respuestas exageradas al mismo (expuestos a estrés crónico como maltrato 0 abuso 0 apegos inseguros) ${ }^{12}$, las manifestaciones se harán persistentemente inadaptadas, tomando los nombres de síntomas psiquiátricos del espectro depresivo-ansioso o de estructuras de personalidad $22,23,34,40$. Esto podría explicar la alta incidencia de la comorbilidad y la espera prudente de la recuperación nutricional antes de decidir la psicofarmacoterapia.

En cuanto al tipo de familia, se trata de familias, en su mayoría, con ambos padres presentes, que se organizan en relación a la adaptabilidad y la cohesión de sus miembros en distintas estructuras familiares ${ }^{26}$. En la muestra, $54,3 \%$ son aglutinadas y rígidas, es decir, los límites relacionales son difusos entre sus miembros y no se adaptan fácilmente a los cambios $^{41,42}$. Los conflictos cambian de cerrados a abiertos en $20,9 \%$, haciéndose posibles de intervenir $^{43}$. Esto constituye un índice de cambio favorable para el trabajo terapéutico, ya que permite el acceso a los patrones emocionales disfuncionales a la base del trastorno de aimentación ${ }^{44,45}$. La familia es tanto un factor de riesgo, como el mejor medio para el tratamiento ${ }^{46-48}$. Esta es la razón por la cual nuestro equipo interviene en las pautas de relación familiar y solicita, desde el inicio, que los padres tomen parte activa en el sistema terapéutico ${ }^{49}$.

Podemos concluir que en esta primera investigación descriptiva del quehacer de un equipo terapéutico en trastornos de alimentación, los resultados a seis meses muestran cambios en el IMC en pacientes anoréxicos restrictivos con rasgos obsesivos de personalidad y que las familias abren su conflicto haciendo más probable su resolución.

Creemos que un modelo coherente de atención basado en la cooperación, comunicación y 
congruencia de las intervenciones nutricionales, psicológicas y relacionales mantiene un sistema terapéutico plástico y sinérgico con capacidad de adaptación y ajuste a cada familia consultante, haciendo de mediador entre el paciente, los cuidados familiares y el trastorno de alimentación. Este fino acoplamiento, en su recurrencia, induciría cambios en los sistemas emocionales de regulación afectiva cambiando de este modo el espacio donde el trastorno de alimentación se

\section{REFERENCIAS}

1. Nielsen S, Moler-Madsen S, Isager T, Jorgensen J, Pagsberg K, Theander S. Standardized mortality in eating disorders: a quantitative summary of previously published and new evidence. J Psychosom Res 1998; 44: 413-34.

2. PatTon GC. Mortality in eating disorders. Psychol Med 1988; 18: 947-51.

3. Crisp AH, Caumender JS, Halek C, Hsu LKG. Longterm mortality in anorexia nervosa: a 20-year follow-up of the St George's and Aberdeen cohorts. Br J Psychiatry 1992; 161: 104-7.

4. Suluvan PF. Mortality in anorexia nervosa. Am J Psychiatry 1995; 152: 1073-4.

5. Steinhausen HC, Rauss-Mason C, Seidel R. Followup studies of anorexia nervosa: a review of four decades of outcome research. Psychol Med 1991; 21: 447-54.

6. Bergh C, Brodin U, Lundberg G, Södersten P. Randomized controlled trial of a treatment for anorexia and bulimia nervosa. Proc Natl Acad Sci USA 2002; 99: 9486-91.

7. Ріснот P. Manual diagnóstico y estadístico de los trastornos mentales DSMIV Masson, S:A Barcelona, 1995.

8. CoRDELLA P. Proposición de un modelo para comprender la configuración anoréctica. Rev Chil Pediatr 2002; 73: 566-75.

9. Bowıy J. Vínculos afectivos: formación, desarrollo y pérdida. Ed Morata Madrid, 1976.

10. WinNICOTT. La natuleza humana. Paidos Buenos Aires, 1996.

11. Garfinkel PE, Garner DM. Anorexia nervosa: A Multidimensional Perspective. New York, NY: Brunner/Mazel. 1982. alojaría en el psiquismo de los pacientes y en la estructura familiar.

Este estudio no da cuenta del seguimiento a largo plazo de su población, ni compara un modelo de intervención familiar-dinámico con otros. Los futuros desafíos incluyen aislar variables de pronóstico y seguimiento, que nos permitan reconocer gravedad y curso del cuadro precozmente con el fin de seleccionar las mejores formas de intervenir, mejorando la calidad de vida a largo plazo.

12. NeMEROFF CB. The Neurobiology of Depression. Sci Am 1998; 278: 42-9.

13. Selvini M. Ragazze. Anoressiche e Bulimiche. Milano. Raffaello Cortina Editore 1998.

14. StierLin H, WeBer G. Que hay detrás de la puerta de la familia: llaves sistémicas para la apertura, comprensión y tratamiento clínico de la anorexia nerviosa. Barcelona. Gedisa 1997.

15. Stein D, Lilenfeld LR, Plotnicov K, Poшice C, Rao R, Strober M et aL. Familial aggregation of eating disorders: results from a controlled family study of bulimia nervosa. Int J Eat Disord 1999; 26: 211-5.

16. Behar R, De la Barrera M, Michelotti J. Identidad de género y trastornos de la conducta alimentaria. Rev Méd Chile 2001; 129: 1003-11.

17. Caparrós N. La anorexia, una locura del cuerpo. Madrid. Biblioteca Nueva, 1997.

18. Halmi KA, Eckert E, Marchi P, Sampugnaro V, Apple $\mathrm{R}$, Conen J. Comorbidity of psychiatric diagnoses in anorexia nervosa. Arch Gen Psychiatry 1991; 48: $712-8$.

19. Silva JR, Pizzagali DA, Larson CL, Jackson DC, DAVIDSON RJ. Frontal brain asymmetry in restrained eaters. J Abnorm Psychol 2002; 111: 676-81.

20. Sodersten P, Bergh C, AMmar A. Anorexia nervosa: towards a neurobiologically based therapy. Eur J Pharmacol 2003; 480: 67-74.

21. Patton GC, Seizer R, Caffey C, Carlin JB, Wolfe R. Onset of adolescent eating disorders: population based cohort study over 3 years. BMJ 1999; 318 (7186): 765-8.

22. American Psyquiatric Association Work Group on EAting Disorders. Practice guideline for the treatment of patients with eating disorders (revision). Am J Psychiatry 2000; 157 (1 Suppl): 1-39. 
23. Group of the National Institute for Clinical Excelience \& National Collaborating Centre for Mental HEALTH. Eating disorders. Core interventions in the treatment and management of anorexia nervosa, bulimia nervosa and related eating disorders. Clinical Guideline 9, January 2004. London: Lima Graphics Ltd.

24. Russell GF, Szmukier GI, Dare C, Eisler I. An evaluation of family therapy in anorexia nervosa and bulimia nervosa. Arch Gen Psychiatry 1987; 44: 1047-56.

25. Vandereycken W, Castro J, Vanderlinden J. Anorexia y Bulimia: La familia en su génesis y tratamiento. Martínez Roca, Barcelona. 1991.

26. FaIRBuRn CG, HarRison PJ. Eating disorders. Lancet 2003; 361: 407-16.

27. GARDNER DM. Eating Disorders Inventory-2: Professional Manual. Odessa, Florida, Psychological Assessment Resources, London 1991.

28. Ben-Tovin DI, Walter K, Gilchrist P. Outcome in patients with eating disorders: a 5 year study. Lancet 2001; 357: 1254-58.

29. Burrows R, Muzzo S. Estándares de crecimiento y desarrollo del escolar chileno. Rev Chil Nutr 1999; 26 (S1): 113-20.

30. Olson DH, Russell CS, Sprenkie DH. Circumplex Model of Marital and Family Systems: VI. Theoretical update. Fam Process 1983; 22: 69-83.

31. Behar R, De la Barrera M, Michelotti J. Feminidad, masculinidad, androginidad y trastornos del hábito del comer. Rev Méd Chile 2002; 130: 964-75.

32. Keel PK, Dorer DJ, Eddy KT, Delinsky S, Franko DL, BLAIS MA ET AL. Predictors of treatment utilization among women with anorexia and bulimia nervosa. Am J Psychiatry 2002; 159: 140-2.

33. Mora F. El cerebro íntimo: ensayos sobre neurociencia. Ariel, Barcelona Neurociencia. 1996.

34. Lock MD. Can family-based treatment of anorexia nervosa be manualized? J Psychotherapy Pract 2001; 10: 253-61.

35. Westen D, Harnden-Fisher J. Personality profiles in eating disorders: Rethinking the distinction between axis I and axis II. Am J Psychiatry 2001; 158: 547-62.

36. Muzzo S, Cordero J, Burrows R. Cambios en la prevalencia del exceso de peso del escolar chileno en los últimos ocho años. Rev Chil Nutr 1999; 26: 311-5.
37. Ben-Tovim DI, Walker K, Gilchrist P, Freeman R, Kalucy R, Esterman A. Outcome in patients with eating disorders: a 5-year study. Lancet 2001; 357: 1254-57.

38. Halmi KA, Sunday SR, Strober M, Kaptan A, Woodside DB, Fichter M ET AL. Perfectionism in anorexia nervosa: variation by clinical subtype, obsessionality, and pathological eating behaviour. Am J Psychiatry 2000; 157: 1799-805.

39. Button EJ, Sonuga-Barke EJ, Davies J, Thompson M. A prospective study of self-esteem in the prediction of eating problems in adolescent schoolgirls: questionnaire findings. Br J Clin Psychol 1996; 35: 193-203.

40. Joel Y. Practice Guideline for the treatment of patients with eating disorders. Am J Psychiatry 2000; 157: 1753-4.

41. Hercovici C. Anorexia y bulimia. Paidos, Buenos Aires. 1998.

42. Minuchen S. Familias y terapia familiar. Paidos, Buenos Aires. 1982.

43. North C, Gowers S, Byram V. Family functioning and life events in the outcome of adolescent anorexia nervosa. Br J Psychiatry 1997; 171: 545-9.

44. Fairburn CG, COOPER Z, DoL HA, WeLCh SL. Risk factors for anorexia nervosa: three integrated case-control comparisons. Arch Gen Psychiatry 1999; 56: 468-76.

45. Golden NH, Katzman DK, Kreipe RE, Stevens SL, SAWYer SM, ReEs J ET al. Society for Adolescent Medicine. Eating disorders in adolescents: Position paper of the Society for Adolescent Medicine. J Adolesc Health 2003; 33: 496-503.

46. Eisler I, Dare C, Russell GF, Szmukier G, ie Grange D, Dodge E. Family and individual therapy in anorexia nervosa. A 5-year follow-up. Arch Gen Psychiatry 1997; 54: 1025-30.

47. Le Grange D. Evaluation of family treatments in adolescent anorexia nervosa: a pilot study. Int J Eat Disord 1992; 12: 347-58.

48. Steinhausen HC. The outcome of anorexia nervosa in the 20th century. Am J Psychiatry 2002; 159: 1284-93.

49. Dare C, EISLER I. Multi-family Group Day Treatment Programme for Adolescent Eating Disorder. Eur Eat Disorders Rev 2000; 8: 4-18. 